\title{
Nouveau modèle analytique pour une meilleure estimation des flux nets annuels en métaux dissous. Cas du cadmium dans l'estuaire de la Gironde
}

\section{New computation for a better estimation of the annual dissolved metal net fluxes. The case of the cadmium in the Gironde estuary}

\author{
F. Pougnet ${ }^{1}$, G. Blanc ${ }^{1, a}$, E. Mulamba-Guilhemat ${ }^{2, b}$, A. Coynel $^{1}$, \\ T. Gil-Diaz ${ }^{1}$, C. Bossy ${ }^{1}$, E. Strady ${ }^{3, b}$, J. Schäfer ${ }^{1}$ \\ 1 UMR 5805 EPOC, Université Bordeaux/CNRS, Allée Geoffroy Saint Hilaire Bat B18N, CS 50023, \\ 33615 Pessac Cedex, France gerard.blanc@u-bordeaux.fr \\ 2 Onepoint, 28 avenue Léonard de Vinci, 33600 Pessac, France \\ 3 Université de Grenoble Alpes, CNRS, IRD, Grenoble INP, IGE, 38000 Grenoble, France
}

\begin{abstract}
Résumé - Malgré d'importants efforts d'aménagement initiés en 1987 et 2007 et la diminution consécutive des émissions issues du bassin versant de la Gironde, les teneurs en cadmium $(\mathrm{Cd})$ présentent dans les huîtres de l'aval de la Gironde restent supérieures à la norme de consommation ( $5 \mathrm{mg} / \mathrm{kg}$, p.s., CE). Des concentrations en cadmium dissous ont été mesurées dans les eaux de surface collectées dans l'estuaire de la Gironde au cours de neuf campagnes océanographiques (2014-2015). Les concentrations théoriques en cadmium dissous $\left(\mathrm{Cd}_{d}{ }^{0}\right)$ et les flux nets journaliers de $\mathrm{Cd}_{d}$ ont été obtenus, selon la méthode de Boyle. Ils confirment une diminution de la pollution en $\mathrm{Cd}$ par paliers décennaux, correspondant à celle observée dans les huîtres girondines grâce au réseau national de surveillance des contaminants prioritaires. Cette observation invalidant les précédentes méthodes d'estimation des flux nets annuels, nous proposons un modèle empirique original appliqué aux quatre dernières décennies et basé sur quatre fonctions exponentielles tronquées. Chaque fonction est représentative d'une situation distincte de la pollution du bassin versant relative aux principaux processus de remédiation entrepris au cours des quatre dernières décennies. Les résultats nous ont permis de proposer un abaque de quantification des flux nets annuels de $\mathrm{Cd}_{d}$ utilisant uniquement les données de débit moyen annuel. Ainsi, cette étude démontre l'efficacité de la géochimie de terrain pour obtenir une meilleure quantification de l'exportation de polluants dissous et biodisponibles, des bassins continentaux vers l'océan côtier.
\end{abstract}

Mots-clés - estuaire, cadmium dissous, modélisation, flux nets

\footnotetext{
a Auteur à joindre

b Auteur actuelle
} 


\begin{abstract}
Despite major management efforts initiated in 1987 and 2007 and decreasing emissions in the Gironde watershed, cadmium (Cd) levels in oysters from the downstream Gironde estuary are still higher to the consumption limit $(5 \mathrm{mg} / \mathrm{kg}, \mathrm{dw}, \mathrm{EC})$. Dissolved Cd concentrations were measured in surface water collected in the Gironde estuary during nine cruises $(2014-2015)$. The obtained theoretical $\left(\mathrm{Cd}_{d}{ }^{0}\right)$ concentrations and the daily net $\mathrm{Cd}$ fluxes, using the Boyle's method, confirm a decadal stepping decrease of the Cd pollution corresponding to that observed in the oysters from the long term national monitoring for priority contaminants at the estuary mouth. As this observation invalidates the previous estimating methods for estimating annual net $\mathrm{Cd}_{d}$ fluxes, we propose an original empirical model applicable for the four last decades. This model is based on four exponential functions with defined boundaries for low water discharges. Each of them is representative of one distinct situation of $\mathrm{Cd}$ pollution in the watershed relative to major remediation processes implemented in the last four decades. These results allow to develop a direct estimation of the annual net $\mathrm{Cd}_{d}$ fluxes only based on the data of the annual mean discharge. Thus, this study demonstrates the strength of the field research in geochemistry to obtain a better quantification of the exportation of dissolved bioavailable pollutants from the continental watersheds to the coastal ocean.
\end{abstract}

Keywords - estuary, dissolved cadmium, computation, net fluxes

\section{Introduction}

À l'interface continent-océan sont distingués les flux bruts $\left(F_{\text {bruts }}\right)$, c'està-dire les flux entrants dans l'estuaire au point amont de la marée dynamique et les flux nets ( $\left.F_{\text {nets }}\right)$, c'est-à-dire les flux exportés dans le domaine marin. Pour un élément non conservatif (influencé par la réactivité estuarienne) comme le cadmium ( $\mathrm{Cd}$ ), ces flux ne sont pas égaux. De plus, la détermination de $F_{\text {net }}$ d'un estuaire est plus délicate, principalement en raison de l'inversion des courants (flot/jusant) et du mélange de deux masses d'eau (fluviale/marine).

La quantification de l'exportation en zone côtière des métaux dissous, et notamment du cadmium dissous $\left(\mathrm{Cd}_{\mathrm{d}}\right)$, utilise la méthode de Boyle et al. (1974, 1982) qui permet l'estimation d'un flux net journalier. Cette méthode nécessite l'acquisition au cours d'un cycle de marée de données de concentrations métalliques dissoutes sur la totalité du gradient de salinité estuarien. Cette acquisition de terrain est seule capable de décrire le comportement non conservatif des métaux dissous dans les eaux intermédiaires et de déterminer la pente et l'ordonnée à l'origine de la droite de dilution résultant du mélange physique entre l'eau de mer et les eaux douces. Selon la méthode de Boyle et al. (1974), cette ordonnée à l'origine est la concentration métallique à salinité zéro dont le produit avec le débit de la rivière donne une valeur du flux net journalier (en kg/jour). Toutefois, la justesse des valeurs de $F_{\text {nets }}$ dissous annuels métalliques reposent essentiellement sur la connaissance de la variabilité des concentrations métalliques à salinité zéro $\left(\mathrm{C}^{0}\right)$ en fonction des débits. Cette variabilité temporelle au sein d'un système estuarien macrotidal comme l'estuaire de la 
Gironde introduit une difficulté majeure dans la détermination des flux nets annuels de métaux dissous. Ainsi cette étude propose une méthode de quantification des flux nets annuels de métaux dissous plus précise que celle précédemment publiée (e.g. Chester, 1990 ; Dabrin et al., 2009). L'utilisation de la nouvelle méthode de calcul permettra de décrire plus précisément l'évolution des flux nets annuels de cadmium dissous de 1989 à 2017, c'est-à-dire sur la majeure partie de la période de décroissance de la pollution cadmiée en Gironde. L'impact et les modes de la pollution cadmiée en Gironde ont été décrits par les travaux de I'IFREMER (cf. Boutier et Chiffoleau, 1986; Boutier et al., 1989; Claisse et al., 1992; rapports RNO/ $\mathrm{ROCCH}, 2016)$ et de nombreuses publications originales (e.g. Kraepiel et al., 1997; Blanc et al., 1999; Audry et al., 2004a, b; Robert et al., 2004; Audry et al., 2005, 2007a, b; Dabrin et al., 2009, 2014; Larrose et al., 2010, Strady et al., 2011a, b). Ces travaux montrent que trente ans après l'arrêt de la métallurgie du zinc aveyronnaise, cause principale de la pollution cadmiée en Gironde, les teneurs en Cd dans le corps mou des huitres sauvages girondines restent au dessus de la norme de consommation et celles de Marennes-Oléron ne montrent pas une franche diminution. Ainsi cette pollution reste d'actualité, et une meilleure quantification des flux nets annuels de cadmium dissous $\left(F^{\text {an }}{ }_{\text {nets }} \mathrm{Cd}_{d}\right)$ est nécessaire pour démontrer la vergence de la résilience de l'écosystème girondin vis-à-vis de cette pollution qui impacte encore la conchyliculture de Marennes-Oléron.

\section{Matériels et méthodes}

Pour atteindre l'objectif de modélisation analytique des flux nets métalliques, il est impératif d'obtenir un échantillonnage représentatif des situations hydrologiques de l'estuaire (débits d'étiage sévère à forte crue, coefficients de marée de morte et vive eau) sur une période relativement courte pour s'affranchir de facteurs forçant externes (dérive climatique et variation d'activités anthropiques au sein du bassin versant). Pour cela, deux types de programmes d'observations ont été sollicités entre mars 2014 et novembre 2015 (Tab. I).

Les eaux estuariennes ont été collectées à l'aide de bouteilles "Niskin » au cours de trois campagnes océanographiques MGTS (Métaux Gironde Transferts et Spéciation) et lors de 6 campagnes océanographiques SOGIR (Service d'Observation de la Gironde de I'OASU, Observatoire Aquitain des Sciences de l'Univers). Les campagnes MGTS ont été réalisées à bord du "Thalia», navire IFREMER (Institut français de recherche pour l'exploitation de la mer) intégré dans la flotte océanographique française. Les campagnes SOGIR sollicitent le navire "Côtes de la Manche» de la flotte côtière de l'INSU (Institut national des sciences de l'univers). Les campagnes MGTS ont permis un prélèvement dynamique de l'ensemble du gradient de salinité, du point kilométrique $0(\mathrm{PKO})$ à Bordeaux à $\mathrm{PK} 110$ en zone côtière (Fig. 1). Les campagnes SOGIR ont échantillonné le gradient salin de 15 à 30 à PK52, PK 67 et PK 86. 
Tableau I. Synthèse des missions effectuées sur l'estuaire de la Gironde entre mars 2014 et novembre 2015.

Table 1. Summary of the hydrological conditions during MGTS and SOGIR cruises between March 2014 and November 2015.

\begin{tabular}{lllcc}
\hline Mission & Date & Débit $\left(\mathrm{m}^{3} / \mathrm{s}\right)$ & Coefficient de marée & Conditions hydriques \\
\hline MGTS 1 & mars 2014 & 1203 & $93-92$ & Décrue \\
MGTS 2 & mars 2015 & 3450 & $67-79$ & Crue \\
SOGIR 1 & avril 2015 & 670 & $46-47$ & Débits moyens \\
SOGIR 2 & mai 2015 & 779 & $56-60$ & Débits moyens \\
SOGIR 3 & juin 2015 & 358 & $72-72$ & Étiage \\
SOGIR 4 & juillet 2015 & 163 & $84-84$ & Étiage \\
SOGIR 5 & août 2015 & 179 & $67-62$ & Étiage \\
MGTS 3 & octobre 2015 & 248 & $75-85$ & Étiage \\
SOGIR 6 & novembre 2015 & 210 & $61-53$ & Étiage \\
\hline
\end{tabular}

Pour s'affranchir des problèmes de contamination parasite, la vaisselle utilisée du prélèvement à l'analyse des métaux dissous est en polypropylène $(\mathrm{PP})$, préalablement nettoyée avec un détergent chauffé $\left(\mathrm{RBS}^{\oplus}\right.$; LABWASH pure) pendant $1 \mathrm{~h}$, puis décontaminée dans un bain d'acide nitrique $10 \%\left(\mathrm{HNO}_{3}\right.$, Baker Analysed; $M=63,01 \mathrm{~g} / \mathrm{mol}$ ) pendant $72 \mathrm{~h}$ et rincée soigneusement à l'eau ultra-pure (Milli-Q ${ }^{\infty}, 18,2 \mathrm{M} \Omega$, Millipore) comme préconisé dans les études antérieures (e.g. Masson et al., 2006; Dabrin et al., 2009; Lanceleur et al., 2011a). Les échantillons d'eau pour l'analyse de cadmium dissous $\left(\mathrm{Cd}_{\mathrm{d}}\right)$ sont conditionnés par une filtration à $0,2 \mu \mathrm{m}$ sur filtre seringue $(\varnothing 22 \mathrm{~mm})$ en acétate de cellulose (Sartorius ${ }^{\circledR}$ ). Les filtrats sont récupérés dans des flacons en PP de $60 \mathrm{ml}$ décontaminés. Les échantillons sont acidifiés à $\mathrm{I}^{\prime} \mathrm{HNO}_{3}\left(0,5 \%\right.$, PlasmaPur Ultrex $\left.{ }^{\circledR}\right)$ et conservés à $4^{\circ} \mathrm{C}$ jusqu'à analyse. L'analyse de $\mathrm{Cd}_{d}$ a été réalisée sur un ICP-MS Xserie2, Thermo Scientific après une étape de pré-concentration nécessaire pour s'affranchir des interférences dues à la matrice saline des eaux salines. Cette étape est une extraction liquide/solide utilisant la cartouche DigiSEP Bleu (SCP Science) selon un protocole préalablement établi par Dabrin et al. (2009) et Strady et al. (2009). La justesse des analyses et le rendement des techniques d'extraction de la matrice saline ont été contrôlés par l'analyse des matériaux de références certifiés: SLEW- ${ }^{\circledR}$ (eaux d'estuaire) et CASS $-5^{\oplus}$ (eau de mer côtière). Leur analyse durant cette étude montre une justesse de mesure comprise dans l'incertitude du certifié et un rendement d'extraction de $\mathrm{Cd}$ supérieur à $95 \%$ (Tab. II).

\section{Résultats}

\subsection{Les concentrations en cadmium dissous $\left(\mathrm{Cd}_{d}\right.$ en $\left.\mathrm{ng} / \mathrm{l}\right)$}

La distribution des concentrations en $\mathrm{Cd}_{d}$ pour les trois campagnes présente une forme en cloche, avec des maxima de concentrations 


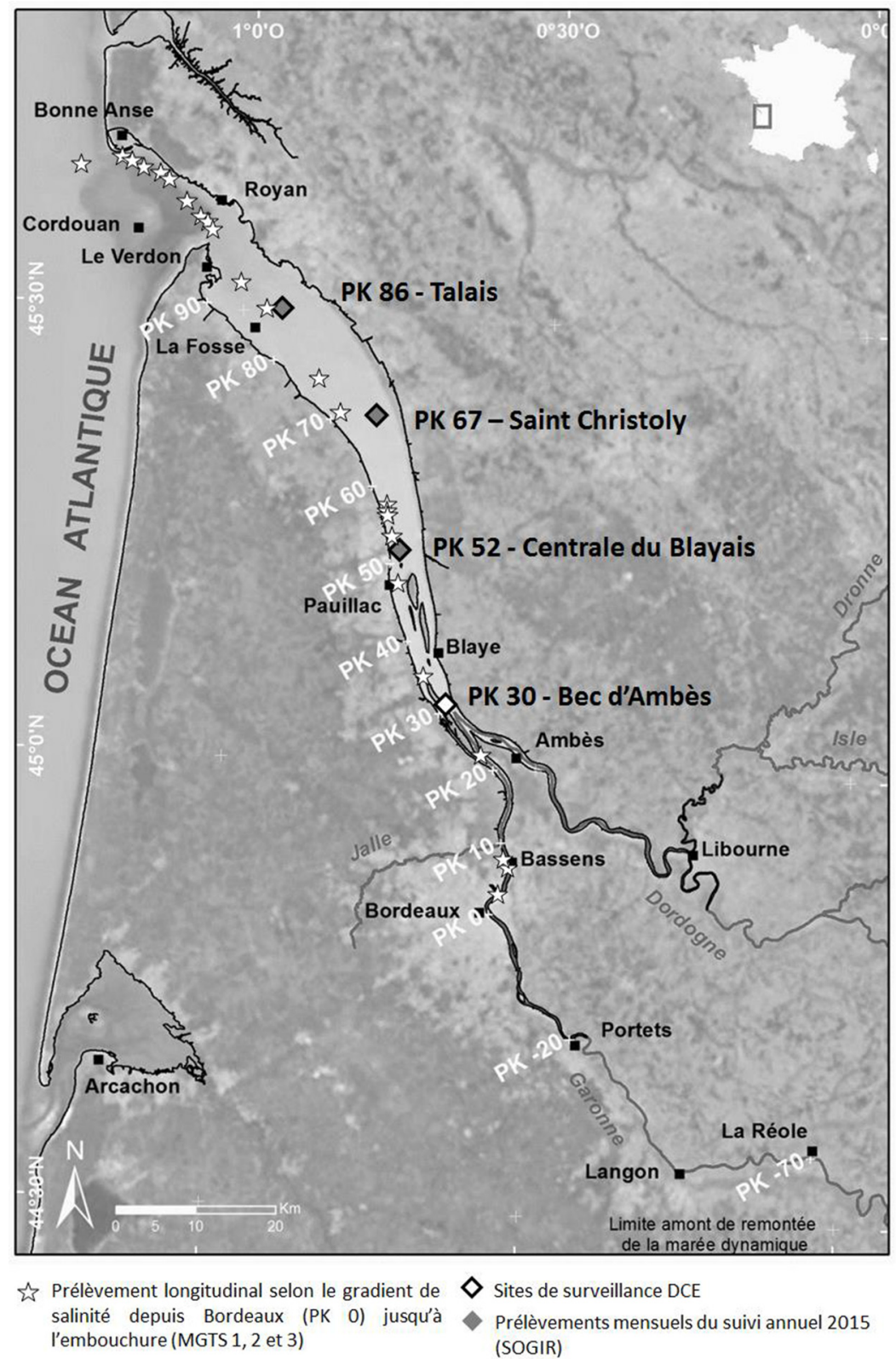

Fig. 1. Carte des sites de prélèvement lors des campagnes MGTS et SOGIR. Les points kilométriques (PK) représentent la distance depuis Bordeaux (PK0).

Fig. 1. Samples location during MGTS et SOGIR cruises. The kilometric points (PK) measure the distance from Bordeaux (PK 0). 
Tableau II. Contrôle qualité et limite de détection (LD) de l'extraction solide/liquide de Cd dissous. Table 2. Dissolved cadmium concentrations of two certified standards and detection limit (LD).

\begin{tabular}{lccc}
\hline & CASS-5 $(\mathrm{ng} / \mathrm{l} ; n=26)$ & SLEW-3 $(\mathrm{ng} / \mathrm{l} ; n=6)$ & LD $(\mathrm{ng} / \mathrm{l} ; n=22)$ \\
\hline Valeur certifiée & $21,5 \pm 1,8$ & $48 \pm 4$ & 3,2 \\
Valeur mesurée & $21,0 \pm 2,8$ & $47 \pm 3$ & \\
Rendements & $98 \%$ & $97 \%$ & \\
\hline
\end{tabular}

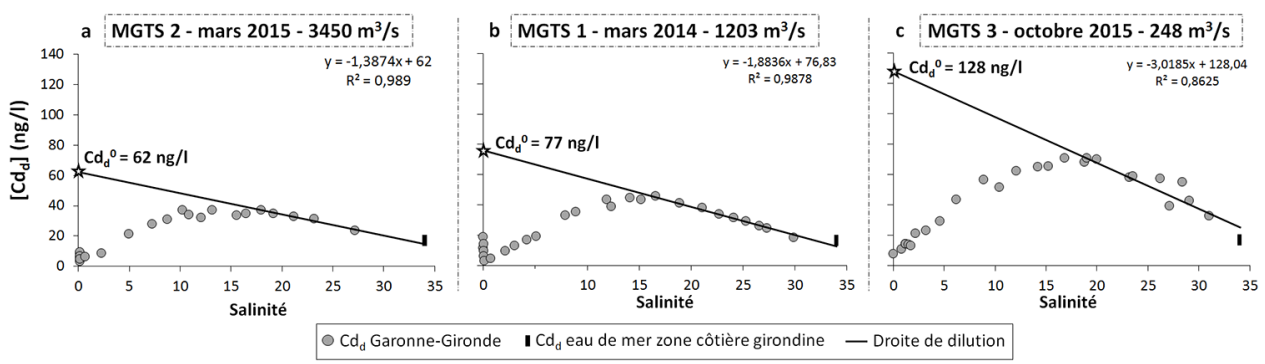

Fig. 2. Distribution des concentrations en $\mathrm{Cd}_{d}$ dans le gradient de salinité pour les trois missions MGTS 1 à 3 . Les $\mathrm{Cd}_{d}{ }^{0}$ sont déterminées à l'aide des régressions linéaires représentant la dilution des eaux estuariennes par les eaux marines.

Fig. 2. Diagram showing the dissolved cadmium concentrations versus the salinity during the MGTS cruises. The linear regressions are applied to obtain the most probable intercept at zero salinity $\left(\mathrm{Cd}_{d}{ }^{0}\right)$ following the method of Boyle et al. (1974, 1982).

$\left(\mathrm{Cd}_{\mathrm{d}} \max \right)$, de 37,46 et $71 \mathrm{ng} / \mathrm{l}$ pour respectivement MGTS2, MGTS 1 et MGTS 3 (Fig. 2), obtenues pour une gamme de salinité moyenne, variant entre 15 et 20. Au-delà des maxima, les concentrations en $\mathrm{Cd}_{d}$ diminuent linéairement vers l'aval, en suivant la droite de mélange physique avec les eaux marines et tendent vers une valeur comprise entre 15 et $20 \mathrm{ng} / \mathrm{l}$. La valeur de $15 \mathrm{ng} / \mathrm{L}$ est déterminée dans les eaux côtières à salinité 33 lors de missions océanographiques réalisées entre 2001 et 2009 en Gironde (Dabrin et al., 2009 ; Strady et al., 2011a). La compilation des concentrations de $\mathrm{Cd}_{\mathrm{d}}$ mesurées en zone côtière entre 1991 et 2006 donne une valeur de $20 \mathrm{ng} / \mathrm{L}$ (Boutier et al., 2000 ; Blanc et al., 2019). Les valeurs de $\mathrm{Cd}_{d}$ max restent, néanmoins largement en dessous de la Norme de Qualité EnvironnementaleMoyenne Annuelle (NQE-MA) de 200 ng/l, fixée par la Directive 2013/ 39/UE pour les eaux marines ou de transition. Cette NQE-MA ne semble pas applicable à des masses d'eau baignant des zones de conchyliculture, puisque les huîtres sauvages de Gironde restent au dessus de la norme de consommation fixée à $5 \mathrm{mg} / \mathrm{kg}$ p.s. par la règlementation (CE) $n^{\circ} 466 / 2001$ du 08/03/2001. Ainsi, le processus naturel d'addition de $\mathrm{Cd}_{d}$ le long du gradient de salinité agit comme une source de $\mathrm{Cd}_{d}$ biodisponible dans les eaux estua- 
riennes et dans les eaux côtières durant les périodes de dessalure lors des forts débits hivernaux et printaniers (Strady et al., 2011a).

\subsection{Les concentrations théoriques en cadmium dissous à salinité zéro $\left(\mathrm{Cd}_{\mathrm{d}}{ }^{\mathrm{O}} \mathrm{en} \mathrm{ng} / \mathrm{l}\right)$ selon la méthode de Boyle et al. (1974).}

Cette étude confirme le comportement non conservatif, additif de $\mathrm{Cd}_{d}$ qui augmente la biodisponibilité de Cd pour les organismes aquatiques, dans les environnements macrotidaux tels que l'estuaire de la Gironde (Elbaz-Poulichet et al., 1987; Jouanneau et al., 1990; Kraepiel et al., 1997; Boutier et al., 2000 ; Michel et al., 2000 ; Robert et al., 2004 ; Dabrin et al., 2009), l'Amazone (Boyle etal., 1982), l'estuaire de la Seine (Chiffoleau et al., 1994, 1999, 2001), l'estuaire et le panache du Chang Jiang (Edmond et al., 1985), l'estuaire de la Loire (Boutier et al., 1993). Ces résultats attestent de l'importance du débit hydrique face à l'intensité de l'addition de Cd. En effet, l'addition la plus faible est mesurée en période de crue $\left(3450 \mathrm{~m}^{3} / \mathrm{s}\right.$; Fig. 2) et la plus forte en période d'étiage $\left(248 \mathrm{~m}^{3} / \mathrm{s}\right.$; Fig. 2). Cette addition différentielle en fonction des débits conditionne la valeur de la concentration de $\mathrm{Cd}_{d}$ à salinité zéro $\left(\mathrm{Cd}_{\mathrm{d}}{ }^{0}\right)$. Ainsi les valeurs de $\mathrm{Cd}_{\mathrm{d}}{ }^{0}$ (ordonnées à l'origine) et la pente des régressions linéaires varient du simple au double entre forte crue et étiage (Fig. 2). Six profils de concentrations correspondant à la dilution de $\mathrm{Cd}_{\mathrm{d}}$ dans les gammes de salinité 15 à 35 ont été acquis durant les campagnes SOGIR. Ces missions ont permis la détermination de neuf nouvelles concentrations à salinité $0\left(\mathrm{Cd}_{d}{ }^{0}\right)$, selon la méthode de Boyle et ainsi de compléter et réactualiser la banque de données préexistantes (Tab. III). Les résultats de $\mathrm{Cd}_{\mathrm{d}}{ }^{0} \mathrm{de}$ 36 campagnes, réalisées sur l'estuaire de 1982 à 2015, sont classés dans le tableau III par gamme de débits (étiage, moyen et crue). Les $\mathrm{Cd}_{d}{ }^{0}$ estimées en période de crue $\left(Q>1000 \mathrm{~m}^{3} / \mathrm{s}\right.$; pendant ou quelques jours après le passage de la crue) varient de 62 à 504 ng/l. Ces concentrations théoriques sont comprises entre 71 et $1041 \mathrm{ng} / \mathrm{l}$ en débit moyen $\left(500<\mathrm{Q}<1000 \mathrm{~m}^{3} / \mathrm{s}\right)$ et entre 128 et $1070 \mathrm{ng} / \mathrm{l}$ en étiage $\left(\mathrm{Q}<500 \mathrm{~m}^{3} / \mathrm{s}\right)$. Avec des concentrations théoriques comprises entre 62 et $77 \mathrm{ng} / \mathrm{l}$ en période de crue, entre 71 et $92 \mathrm{ng} / \mathrm{l}$ en période de débit moyen et entre 128 à $217 \mathrm{ng} / \mathrm{l}$ en période d'étiage, les $\mathrm{Cd}_{\mathrm{d}}{ }^{0}$ déterminées avec les données des campagnes MGTS et SOGIR obtenues entre 2014 et 2015 sont les plus faibles depuis 1982. Ainsi, les $\mathrm{Cd}_{d}{ }^{0}$ ont diminué ces quatre dernières décennies et il n'y a pas de relation simple entre les $\mathrm{Cd}_{\mathrm{d}}{ }^{0}$ et les débits journaliers.

\section{Discussion}

\subsection{Les flux nets journaliers en cadmium dissous $\left(F^{j}{ }_{\text {nets }} \mathbf{C d}_{d}\right.$ en $\mathrm{kg} / \mathrm{j})$}

Le produit de la concentration théorique $\left(\mathrm{Cd}_{\mathrm{d}}{ }^{0}\right.$ en $\left.\mathrm{ng} / \mathrm{l}\right)$ par le débit de l'estuaire le jour du prélèvement (Qj en $\mathrm{m}^{3} / \mathrm{s}$ ) donne le flux net journalier de cadmium dissous $\left(\mathrm{F}_{\text {nets }}^{\mathrm{j}} \mathrm{Cd}_{\mathrm{d}}\right.$ en $\left.\mathrm{kg} / \mathrm{j}\right)$. Ici Qj Gironde est déduit de la somme des QjGaronne + QjDordogne + Qjlsle, les trois principaux tributaires de l'estuaire de la Gironde. Ainsi, la décroissance temporelle des valeurs de $\mathrm{Cd}_{d}{ }^{0}$ détermine 


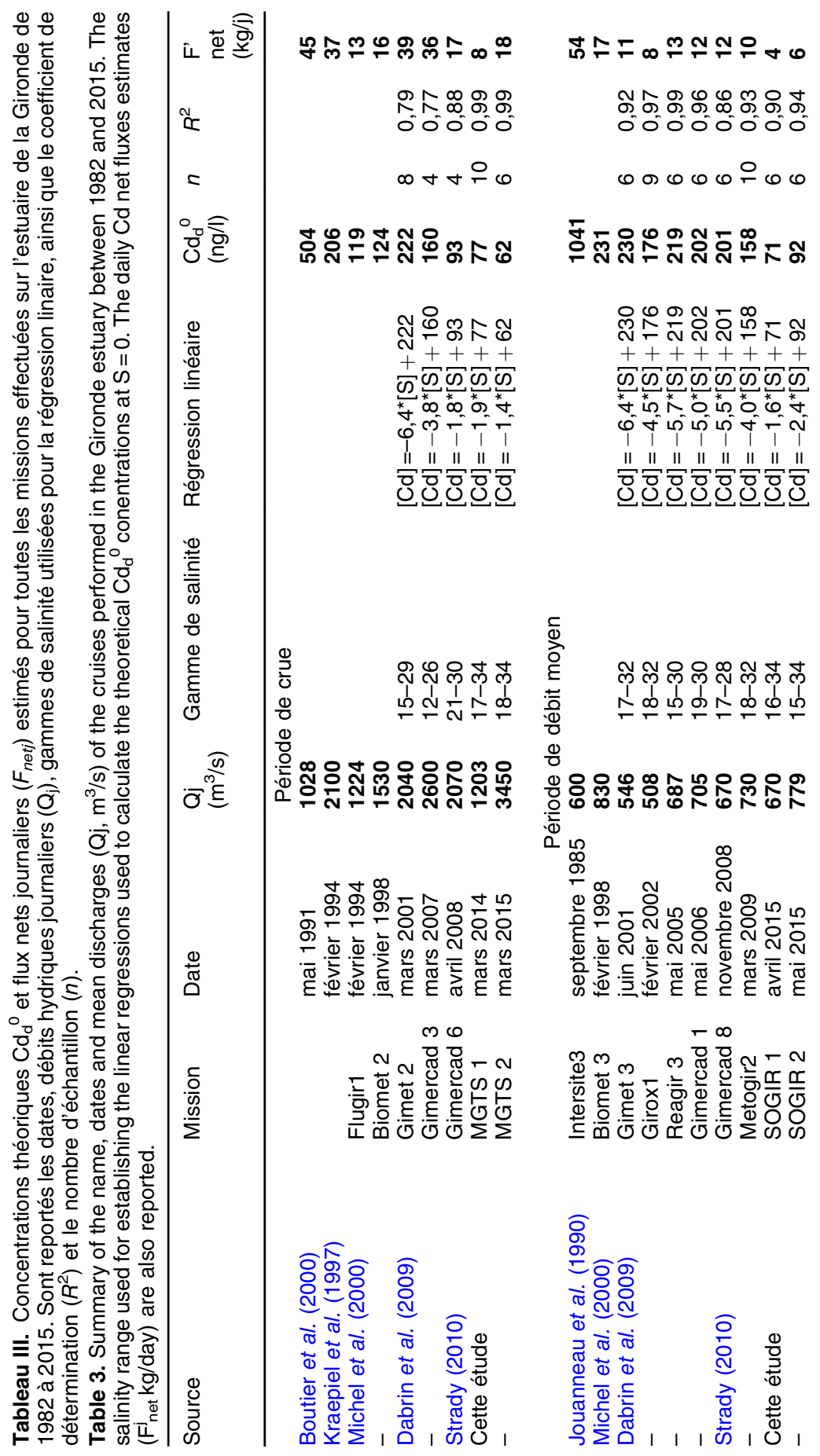




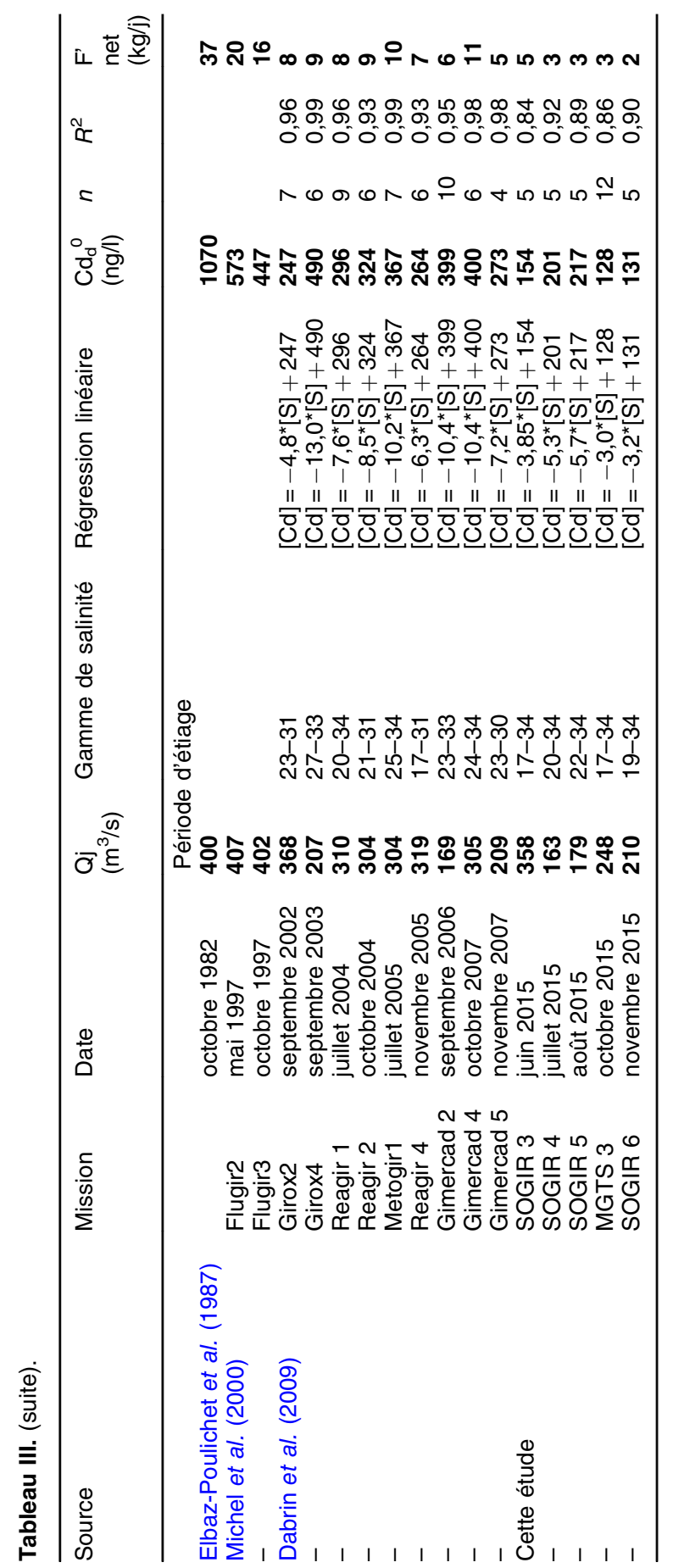




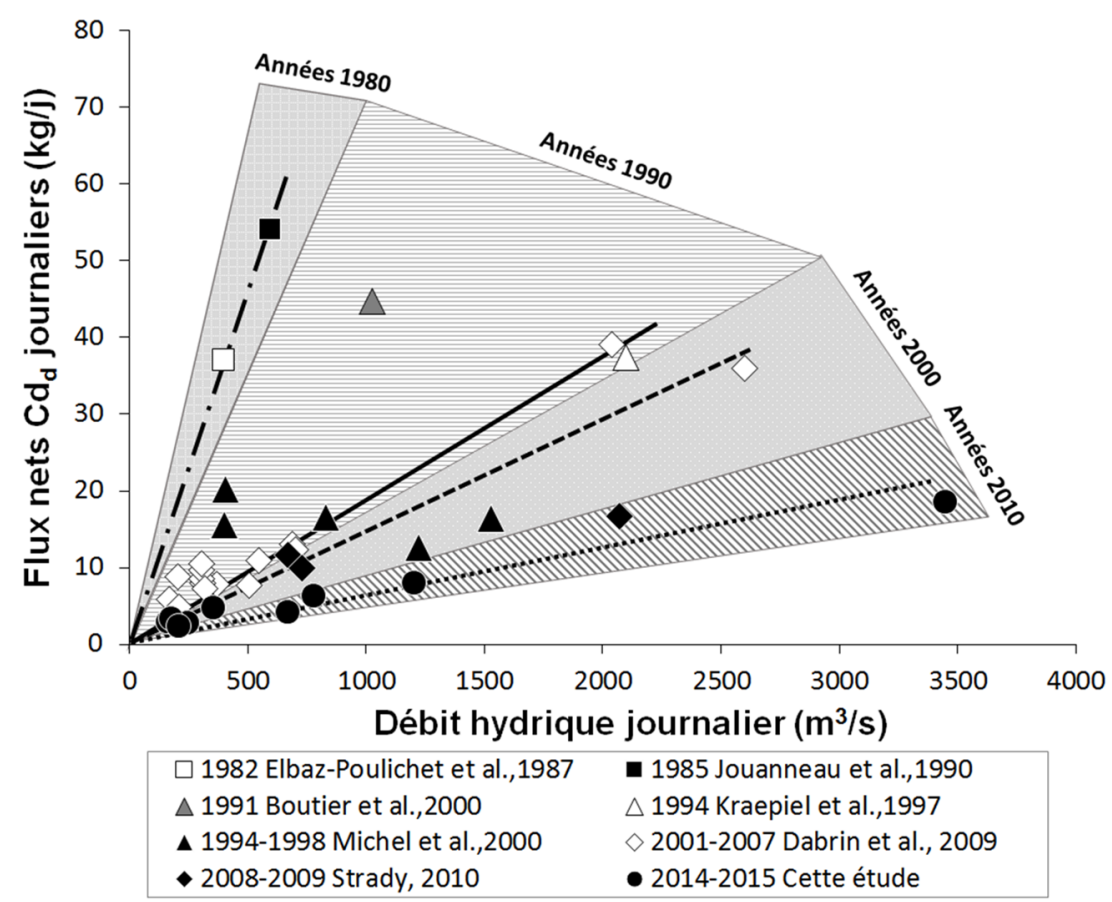

Fig. 3. Flux nets journaliers de cadmium dissous $\left(\mathrm{Cd}_{d}\right)$ en fonction des débits hydriques pour toutes les missions effectuées sur l'estuaire de la Gironde entre 1982 et 2015 (d'après Strady, 2010 modifiée).

Fig. 3. Diagram showing daily Cd net fluxes $\left(\mathrm{kg}\right.$.day $\left.{ }^{-1}\right)$ versus daily discharges $\left(\mathrm{m}^{3} / \mathrm{s}\right)$ during Gironde estuary cruises from 1982 to 2015 (after Strady, 2010, modified).

une diminution des $\mathrm{F}_{\text {nets }}^{\mathrm{j}} \mathrm{Cd}_{\mathrm{d}}$ depuis 1982 comme préalablement constaté entre 1982 et 2010 par Strady (2010) qui proposait une diminution décennale des $\mathrm{F}_{\text {nets }}^{\mathrm{j}} \mathrm{Cd}_{\mathrm{d}}$ (Fig. 3).

Les neuf nouvelles valeurs de $\mathrm{F}_{\text {nets }}^{\mathrm{j}} \mathrm{Cd}_{\mathrm{d}}$ acquises confirment cette tendance décennale à la décroissance. Dans les années 2010, un flux de $18 \mathrm{~kg} / \mathrm{j}$ est obtenu pour un débit supérieur à $3000 \mathrm{~m}^{3} / \mathrm{s}$ (Fig. 3), alors que pour les années 2000 (Michel et al., 2000; Dabrin et al., 2009), des flux similaires sont constatés pour une gamme de débit proche de 1000 à $2000 \mathrm{~m}^{3} / \mathrm{s}$. Pour les années 1990, des flux de 18 à
$20 \mathrm{~kg} / \mathrm{j}$ sont délivrés pour des débits compris entre 500 et $1000 \mathrm{~m}^{3} / \mathrm{s}$ (Kraepiel et al., 1997; Michel et al., 2000). Entre 1982 et 1992, des flux de 35 à $55 \mathrm{~kg} / \mathrm{j}$ sont reportés pour des débits proches de ceux précédemment cités (Elbaz-Poulichet et al., 1987; Jouanneau et al., 1990 ; Boutier et al., 2000). Cette diminution temporelle des $\mathrm{F}_{\text {nets }}^{\mathrm{j}} \mathrm{Cd}_{\mathrm{d}}$ est à mettre en relation avec les activités métallurgiques du zinc de Viviez en Aveyron qui constituent la principale source de cadmium de l'estuaire de la Gironde (Latouche, 1988; Blanc et al., 1999; Schäfer et al., 2002; Schäfer et Blanc, 2002 ; Audry, 2003; 


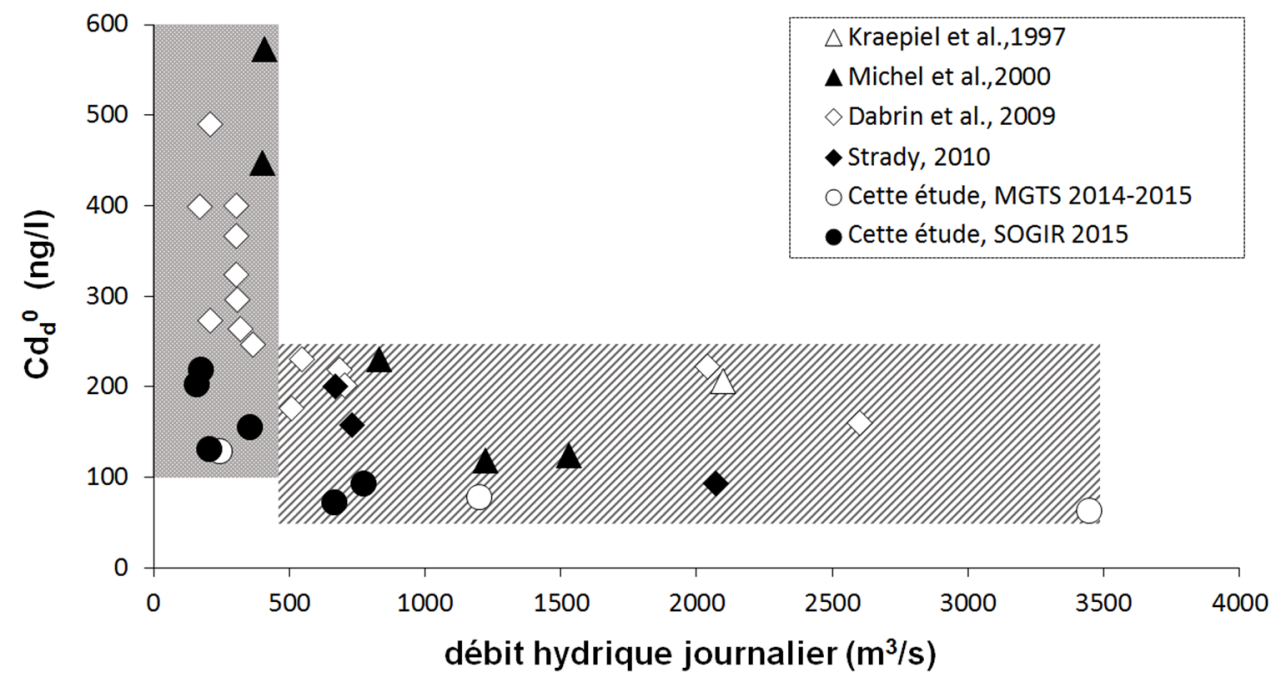

Fig. 4. Compilation des données (1994 à 2015) de concentrations théoriques en cadmium dissous $\left(\mathrm{Cd}_{d}{ }^{0}\right)$ dans l'estuaire de la Gironde en fonction du débit hydrique journalier. Les données de cette étude sont représentées par des cercles noirs et blancs. Le rectangle gris représente la zone $\mathrm{Qj}<500 \mathrm{~m}^{3} / \mathrm{s}$ avec des $\mathrm{Cd}_{d}{ }^{0}$ comprises entre 100 et $600 \mathrm{ng} / \mathrm{l}$. Le rectangle hachuré représente les $\mathrm{Cd}_{d}{ }^{0}$ comprises entre 50 et $250 \mathrm{ng} / \mathrm{l}$ pour des débits moyens à forts (d'après Dabrin et al., 2009, modifié).

Fig. 4. Theoretical $\mathrm{Cd}_{d}{ }^{0}$ concentrations over the whole range of water discharges recorded for the cruises from 1994 to 2015. Black and white circles correspond to the data of this study (2014-2015). Grey area highlights $\mathrm{Cd}_{d}{ }^{0}$ concentrations ranged from 100 to $600 \mathrm{ng} / \mathrm{l}$. Hatched area highlights $\mathrm{Cd}_{\mathrm{d}}{ }^{0}$ concentrations ranged from 50 to $250 \mathrm{ng} / \mathrm{l}$. (after Dabrin et al., 2009, modified).

Audry et al., 2004a; Blanc et al., 2006). Les séries sédimentaires fluviales et estuariennes ont enregistré l'évolution temporelle de la pollution cadmiée en fonction de l'augmentation de la production industrielle depuis les années 1950, de l'arrêt de la métallurgie en 1987, et de la première étape de remédiation de site industriel finalisée en 1994 (Grousset et al., 1999; Audry et al., 2004c; Larrose et al., 2010). Ainsi, les $\mathrm{F}_{\text {nets }}^{\mathrm{j}} \mathrm{Cd}_{d}$ dépendent à la fois des débits hydriques et de la pression anthropique résultant principalement des rejets de $\mathrm{Cd}$ industriel du bassin versant de Decazeville. Sachant que le cadmium anthropique est à plus de $98 \%$ transporté dans la rivière adsorbé sur les particules (Blanc et al., 1999; Schäfer et al., 2002; Schäfer et Blanc, 2002; Audry et al., 2004a) et que ce $\mathrm{Cd}$ anthropique est majoritairement libéré et stabilisé en phase dissoute sous-forme de chloro-complexes dans les eaux intermédiaires et marines (Mantoura et al., 1978; Mantoura, 1981; Comans et van Dijk, 1988), il est normal de constater une forte variabilité des $\mathrm{Cd}_{d}{ }^{0}$. La figure 4 montre une forte variabilité des valeurs de $\mathrm{Cd}_{d}{ }^{0}$ pour des débits inférieurs à $500 \mathrm{~m}^{3} / \mathrm{s}$ (étiage) et une plus faible variabilité pour des débits supérieurs à $500 \mathrm{~m}^{3} / \mathrm{s}$ (débits moyens et crues). Ces variabilités de $\mathrm{Cd}_{d}{ }^{0}$ dépendent évidemment des débits 
qui déterminent également les apports solides dans l'estuaire mais aussi de la charge en $\mathrm{Cd}$ anthropique portée par les particules fluviales. Les temps de résidence des eaux (de 20 à 90 jours) et des particules fluviales (de 20 jours à 3 ans) dans l'estuaire (Castaing et Jouanneau, 1979) agissent de façon asynchrone sur l'addition de $\mathrm{Cd}$ dissous dans l'estuaire. Ainsi, les valeurs obtenues pour les flux nets journaliers de cadmium dissous dérivent de la diminution de la source fluviale et de la réactivité estuarienne lors de la dilution des particules fluviales dans le gradient de salinité. En conséquence, une transposition des flux nets de $\mathrm{Cd}_{d}$ journaliers en flux annuels sur une base d'extrapolation numérique utilisant les banques de données de débits journaliers est une démarche qui donne des résultats crédibles sur l'estimation moyenne des flux annuels mais qui génèrent de fortes marges d'erreurs. Cette démarche était, cependant, jusqu'à présent la plus performante pour estimer la variabilité interannuelle des flux nets de $\mathrm{Cd}_{\mathrm{d}}$ (e.g. Dabrin et al., 2009). Les estimations antérieures des flux nets annuels par simple extrapolation à l'échelle annuelle d'une seule valeur de $\mathrm{Cd}_{\mathrm{d}}{ }^{0}$ impliquent nécessairement de fortes incertitudes (Elbaz-Poulichet et al., 1987 ; Boutier et al., 2000).

\subsection{Les flux nets annuels en cadmium dissous ( $\mathrm{F}_{\text {nets }}^{\text {an }} \mathrm{C} \mathbf{C d}_{\mathbf{d}}$ en t/an)}

Dans le but d'estimer plus précisément l'export interannuel de $\mathrm{Cd}_{d}$ en zone côtière, nous proposons pour la première fois, une démarche utilisant un modèle empirique analytique de calcul de $\mathrm{F}_{\text {nets }}^{\mathrm{an}} \mathrm{Cd}_{\mathrm{d}}$. Le modèle analytique a été construit en utilisant initialement les données acquises entre 2014 et 2015 qui sont nombreuses dans un temps d'observation suffisamment court pour s'affranchir des variations dues à des facteurs forçant autres que le débit. Ce modèle a été ensuite adapté pour rendre compte de la variabilité des données de $\mathrm{Cd}_{\mathrm{d}}{ }^{0}$ acquises antérieurement entre 1982 et 2010. II utilise des techniques mathématiques traditionnelles de type analyse de régression pour développer des équations, incluant des paramètres d'ajustement et décrivant le mieux possible la distribution des données de $\mathrm{Cd}_{d}{ }^{0}$ en fonction des débits. La banque de données d'observation semble suffisante pour que les modèles proposés rendent compte des variations saisonnières de débits fluviaux et de la baisse des apports fluviaux de $\mathrm{Cd}$ industriel depuis 1982.

La détermination des $\mathrm{F}_{\text {nets }}^{\mathrm{an}} \mathrm{Cd}_{\mathrm{d}}$ entre 1982 et 2015 s'appuie sur quatre équations de type exponentielles décroissantes tronquées (Fig.5). Ces équations et leurs paramètres d'ajustement sont reportés dans le tableau IV.

Dans ces paramètres, les variables a et $c$ correspondent aux valeurs de l'ordonnée à l'origine lorsque $y=a e^{b x}$ $\mathrm{ou}=c e^{d x}$, où $y=\mathrm{Cd}_{\mathrm{d}}{ }^{0}$ (concentration théorique à salinité zéro) et $x=Q_{\mathrm{j}}$ (débit hydrique journalier résultant de la sommation des débits des tributaires majeurs de l'estuaire déterminés en amont de la marée dynamique). Les variables $b$ et $d$ sont les vitesses de décroissance relatives de $\mathrm{Cd}_{d}{ }^{0}$ par rapport à $Q_{\mathrm{j}}$. Si $b>d$, cela implique une décroissance de $\mathrm{Cd}_{\mathrm{d}}{ }^{0}$ pour les faibles débits plus importante que pour 


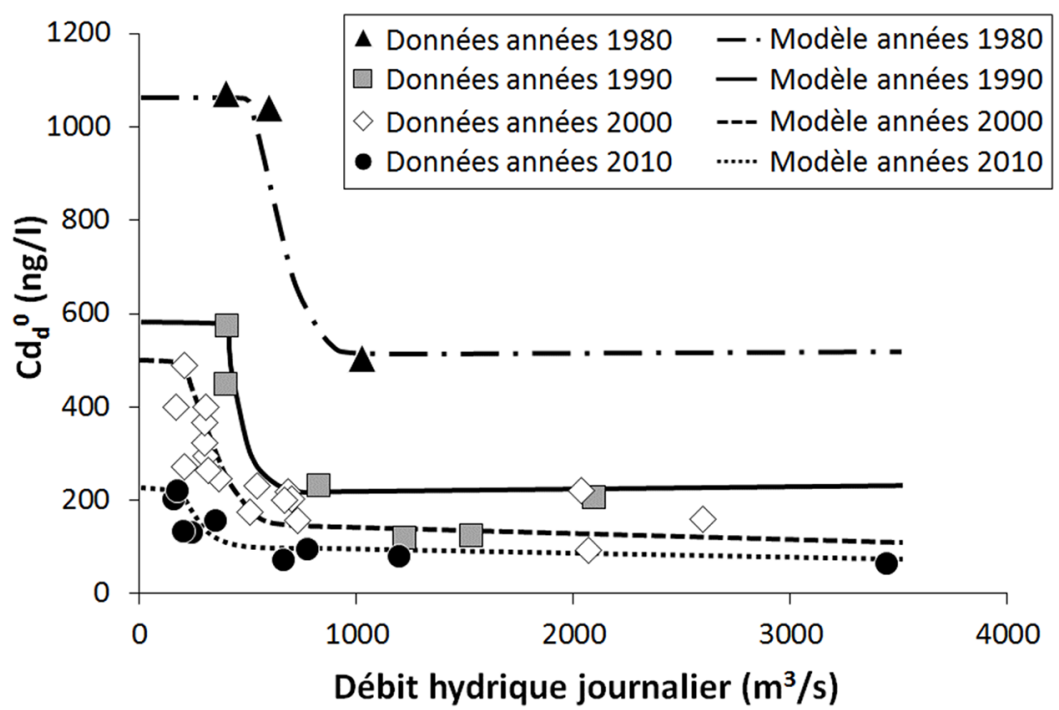

Fig. 5. Diagramme présentant les lignes des fonctions exponentielles tronquées utilisées pour estimer les flux nets annuels de $\mathrm{Cd}$ dissous (t/an). Les cercles noirs correspondent aux données acquises en 2014 et 2015.

Fig. 5. Diagram showing lines of the exponential functions with bounds used for estimation of the annual dissolved Cd net fluxes (t/an) over the four earlier decades. Black circles correspond to the data of this study.

Tableau IV. Paramètres d'ajustement et équations utilisées pour estimer les flux nets annuels de cadmium dissous pour les quatre dernières décennies.

Table 4. Fitting parameters and mathematical functions used to estimate the values of the annual net $\mathrm{Cd}_{d}$ fluxes over the last earlier decades.

\begin{tabular}{lllll}
\hline Décennie & $1980-1990$ & $1990-2000$ & $2000-2010$ & $2010-2016$ \\
\hline Equation & $\begin{array}{c}y=a^{*} \exp \left(b^{*}(x-u)\right)+ \\
c^{*} \exp \left(d^{*}(x-u)\right)+\end{array}$ & $\begin{array}{c}y=a^{*} \exp \left(b^{*} x\right)+ \\
c^{*} \exp \left(d^{*} x\right)\end{array}$ & $\begin{array}{c}y=a^{*} \exp \left(b^{*} x\right)+ \\
c^{*} \exp \left(d^{*} x\right)\end{array}$ & $\begin{array}{c}y=a^{*} \exp \left(b^{*} x\right)+ \\
c^{*} \exp \left(d^{*} x\right)\end{array}$ \\
$C_{d}{ }^{0} \max$ & 1070 & 573 & 490 & 217 \\
$a$ & 136200 & 86870 & 1306 & 445,1 \\
$b$ & $-1,50 \mathrm{E}-02$ & $-1,40 \mathrm{E}-02$ & $-6,01 \mathrm{E}-03$ & $-8,77 \mathrm{E}-03$ \\
$c$ & 203,1 & 201 & 121,1 & 98,07 \\
$d$ & $2,79 \mathrm{E}-05$ & $3,26 \mathrm{E}-05$ & $7,59 \mathrm{E}-06$ & $-1,47 \mathrm{E}-04$ \\
$u$ & 300 & - & - & - \\
$v$ & 250 & - & - & - \\
\hline
\end{tabular}

les hauts débits $\left(Q_{\mathrm{j}}\right)$. La sommation des deux exponentielles donne l'équation: $y=a e^{b x}+c e^{d x}$. Cette sommation permet un bon ajustement des données de $\mathrm{Cd}_{\mathrm{d}}{ }^{0}$ acquises lors des missions de cette étude en 2014 et 2015 mais aussi pour celles des années 1990 et 2000. II est nécessaire d'utiliser 2 équations exponentielles pour représenter les différentes vitesses de décroissance 
entre $\mathrm{Cd}_{\mathrm{d}}{ }^{0}$ et les débits, en deçà et au dessus de $1000 \mathrm{~m}^{3} / \mathrm{s}$. En effet, une seule équation tirerait les $\mathrm{Cd}_{\mathrm{d}}{ }^{0}$ vers l'axe X. Ce choix explique la création de modèles empiriques et à priori non mécanistiques. Néanmoins, d'un point de vue mécanistique, il existe deux cinétiques différentes pour un seul processus de relargage. En effet, 2 vitesses de décroissances sont nécessaires pour expliquer la désorption de $\mathrm{Cd}$ dans l'estuaire, selon le temps de résidence des particules. Exemple: pour de forts $Q(Q>1000$ $\mathrm{m}^{3} \cdot \mathrm{s}^{-1}$ ), la cinétique de désorption est limitée par un temps de résidence des particules plus court par rapport à des conditions d'étiage, impliquant des $\mathrm{Cd}_{\mathrm{d}}{ }^{0}$ plus faibles et peu variables.

Pour les données plus éparses, acquises antérieurement dans les années 1980, l'obtention du meilleur compromis entre la courbe-modèle et les données nécessite la prise en considération des variables $u$ et $v$. Bien que le modèle des années 1980 soit basé sur la mesure de 3 points, nous avons choisi de baser son approximation sur la distribution des $\mathrm{Cd}_{\mathrm{d}}{ }^{0}$ des décennies plus récentes. Ainsi, nous avons privilégié un comportement semblable pour les 4 modèles pour $Q>1000 \mathrm{~m}^{3} \cdot \mathrm{s}^{-1}$. Ce choix implique la création de 2 variables « $u$ » et $« V$ » supplémentaires pour les années 1980, justifiant une forme mathématique différente de celles proposées pour les autres décennies. Ces variables sont nécessaires à l'ajustement des équations exponentielles aux forts $\mathrm{Cd}_{d}{ }^{0}$. Par rapport à l'équation précédente, $u$ modifie la valeur de l'abscisse et $v$ celle de l'ordonnée.
Chaque équation correspond à une borne horizontale représentant les quatre valeurs les plus fortes de $\mathrm{Cd}_{\mathrm{d}}{ }^{0}$ déterminées, respectivement pour chaque décennie et pour $Q_{\mathrm{j}}<500 \mathrm{~m}^{3} / \mathrm{s}$ $\left(\mathrm{Cd}_{\mathrm{d}}{ }^{0} \mathrm{max}\right.$; Tab. III). Le choix de l'ajout d'une troncature se justifie car: (i) par définition, l'équation exponentielle tend naturellement vers l'infini pour de faible débit, ce qui ne semble pas être un comportement cohérent d'un point de vue mécanistique; (ii) des conditions d'étiage extrême n'étant pas représentatives des conditions annuelles de débits sur l'estuaire de la Gironde implique le choix de prendre une valeur constante pour ces faibles débits. De plus, la valeur de cette troncature ne change pas significativement la valeur du flux annuel. En considérant par exemple, des valeurs de $\mathrm{Cd}_{\mathrm{d}}{ }^{0}$ un ordre de grandeur supérieur à la valeur constante choisie pour le modèle décennal (2010), avec une moyenne de jour d'étiage extrême $\left(Q<\sim 150 \mathrm{~m}^{3} . \mathrm{s}^{-1}\right)$ de 25 jours à l'année, l'erreur d'estimation sur les flux nets annuels est de l'ordre du $1 \%$. De plus, la décroissance observée de 1070 à $217 \mathrm{ng} / \mathrm{l}$ pour les $\mathrm{Cd}_{\mathrm{d}}{ }^{0}$ max depuis les années 1980 à 2010 témoigne de la diminution de la pollution cadmiée d'un facteur 5 . Ce taux de décontamination est comparable à la baisse des concentrations en $\mathrm{Cd}$ de 100 à $20 \mathrm{mg} / \mathrm{kg}$ p.s. mesuré dans le corps mou des huîtres sauvages au site d'observation Ifremer RNO-ROCCH «La Fosse » localisé en aval de l'estuaire (Fig. 1). II semble que cette décontamination se fasse par seuils successifs quasi décennaux (Fig. 6) en relation avec les étapes de décontamination du site 


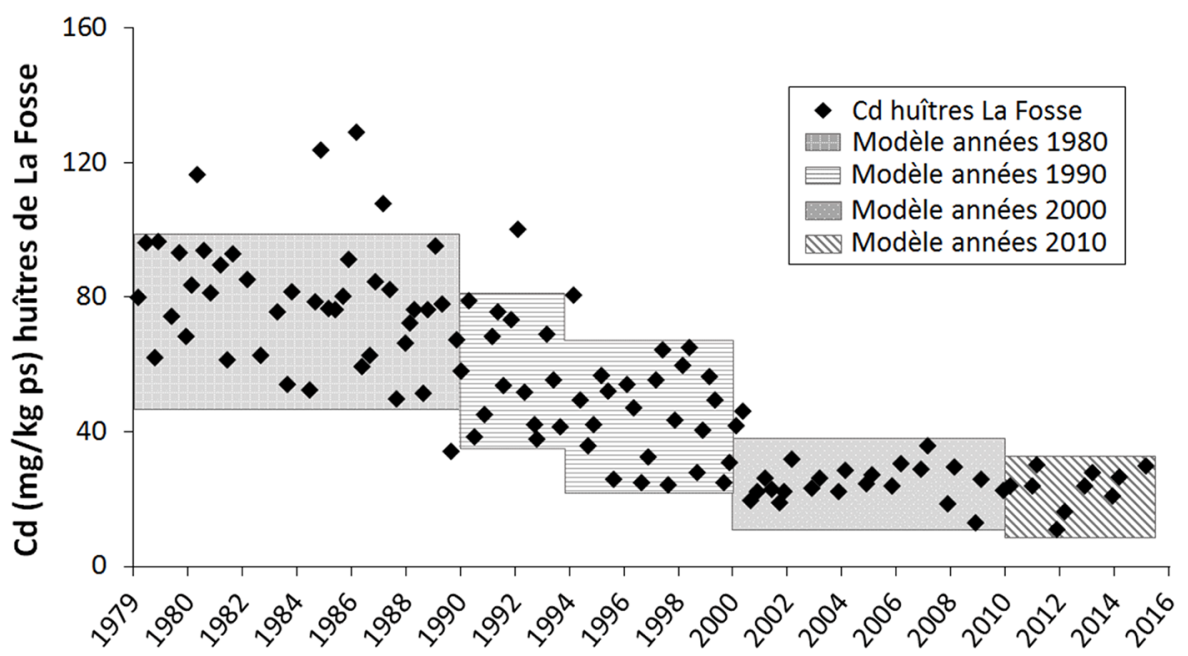

Fig. 6. Concentrations en cadmium (mg/kg p.s.) dans les huîtres creuses (Crassostrea gigas ou nouvellement nommée Magallana Gigas) du site de surveillance La Fosse sur l'Estuaire de la Gironde entre 1979 et 2016 (d'après données du RNO/ROCCH de l'Ifremer). Les rectangles grisés et hachurés montrent une décroissance de la pollution cadmiée par seuils successifs quasi décennaux qui correspondent aux modèles de flux nets utilisés pour les quatre dernières décades.

Fig. 6. Cadmium concentrations (mg/kg d.w.) of the oysters (Crassostrea gigas) from the monitoring "La Fosse" site, downstream Gironde) (after RNO/ROCCH data, Ifremer). Grey and hatched areas show $\mathrm{Cd}$ pollution decreases over the four earlier decades and correspond to the four flux models.

métallurgique du Viviez Aveyron (Audry et al., 2004c; Lanceleur et al., 2011a, b).

La figure 6 permet de formuler I'hypothèse qu'une autre relation $\mathrm{Cd}_{\mathrm{d}}{ }^{0}$ versus débits a pu exister durant la période 1990-1994 correspondant au premier confinement des déchets métallurgiques. En effet, les concentrations en $\mathrm{Cd}$ dans le corps mou des huîtres de La Fosse ont chuté d'environ $25 \%$ entre les périodes 1990-1994 et 1995-2000 (Fig. 6). Ainsi pour rendre compte de cette décontamination en Cd entre 1980 et 2016, chaque courbemodèle a été utilisée pour une seule décennie afin de déterminer les flux de $\mathrm{Cd}_{d}$ nets annuels correspondant $\left(\mathrm{F}_{\text {nets }}^{\mathrm{an}}\right.$ $\left.\mathrm{Cd}_{d}\right)$. Les valeurs des $\mathrm{F}_{\text {nets }}^{\mathrm{an}} \mathrm{Cd}_{\mathrm{d}}$ en $\mathrm{t} / \mathrm{an}$ sont reportées dans le tableau $V$ et sont présentées dans la figure 7 , avec les valeurs des flux nets précédemment publiées et la variation interannuelle des débits hydriques. Pour la détermination de ces valeurs de $\mathrm{F}_{\text {nets }}^{\text {an }} \mathrm{Cd}_{d}$, il ne semble pas justifié de discuter de la robustesse des modèles empiriques proposés puisque ces derniers sont basés sur l'exploitation d'une banque de données unique issue de l'observation pluriannuelle du milieu naturel associées à des contraintes mathématiques (cf. troncature comme limite imposée) nécessaires à la création des équations. Nos valeurs de $\mathrm{F}_{\text {nets }}^{\text {an }}$ $\mathrm{Cd}_{d}$ déterminées entre 2001 et 2007 sont globalement du même ordre de grandeur que les bornes basses de Dabrin et al. (2009). En 1982, la valeur de 14 t/an (Elbaz-Poulichet et al., 1987) est $30 \%$ inférieure à celles données par le présent modèle alors que celle 
Tableau V. Débits moyens annuels $\left(\mathrm{m}^{3} / \mathrm{s}\right)$ avec les résultats des flux nets annuels et leurs moyennes décennales en $\mathrm{Cd}_{d}(\mathrm{t})$. Les rapports des flux nets annuels (t/an) versus flux hydriques annuels $\left(\mathrm{km}^{3} / \mathrm{an}\right)$ sont aussi reportés pour la période 1982 à 2016 comme des concentrations moyennes pondérées (ng/l).

Table 5. Annual mean discharges $\left(\mathrm{m}^{3} / \mathrm{s}\right)$ and calculated annual $\mathrm{Cd}_{d}$ net fluxes showing decadal averages ( $\mathrm{t}$ ). Ratios of the annual $\mathrm{Cd}_{\mathrm{d}}$ net fluxes (t/an) versus the annual discharges $\left(\mathrm{km}^{3} / \mathrm{year}\right)$ are also reported as average discharge-weighted concentrations (ng/l).

\begin{tabular}{|c|c|c|c|}
\hline & $\begin{array}{l}\text { Débit hydrique } \\
\text { annuel moyen }\left(\mathrm{m}^{3} / \mathrm{s}\right)\end{array}$ & $\begin{array}{l}\text { Flux nets } \\
\text { Cdd }_{d}(t / a n)\end{array}$ & $\begin{array}{l}\text { Concentrations moyennes } \\
\text { pondérées (ng/l) }\end{array}$ \\
\hline 1982 & 1016 & 19,8 & 620 \\
\hline 1983 & 829 & 17,1 & 660 \\
\hline 1984 & 948 & 18,3 & 630 \\
\hline 1985 & 881 & 17,3 & 620 \\
\hline 1986 & 871 & 17,3 & 630 \\
\hline 1987 & 818 & 16,4 & 635 \\
\hline 1988 & 1118 & 21,3 & 600 \\
\hline 1989 & 518 & 12,4 & 760 \\
\hline Moy. décennale années 1980 & 875 & 17 & 620 \\
\hline 1990 & 568 & 5,34 & 300 \\
\hline 1991 & 697 & 5,81 & 260 \\
\hline 1992 & 1150 & 8,64 & 240 \\
\hline 1993 & 863 & 6,79 & 250 \\
\hline 1994 & 1305 & 9,45 & 230 \\
\hline 1995 & 906 & 7,31 & 260 \\
\hline 1996 & 1087 & 8,40 & 255 \\
\hline 1997 & 671 & 5,98 & 280 \\
\hline 1998 & 789 & 6,02 & 240 \\
\hline 1999 & 950 & 7,32 & 240 \\
\hline Moy. décennale années 1990 & 899 & 7 & 250 \\
\hline 2000 & 954 & 4,33 & 140 \\
\hline 2001 & 941 & 4,48 & 150 \\
\hline 2002 & 668 & 3,58 & 170 \\
\hline 2003 & 795 & 3,94 & 160 \\
\hline 2004 & 910 & 4,44 & 155 \\
\hline 2005 & 553 & 3,27 & 190 \\
\hline 2006 & 669 & 3,53 & 170 \\
\hline 2007 & 698 & 3,67 & 170 \\
\hline 2008 & 887 & 4,22 & 150 \\
\hline 2009 & 737 & 4,02 & 170 \\
\hline Moy. décennale années 2000 & 781 & 4 & 160 \\
\hline 2010 & 789 & 2,22 & 89 \\
\hline 2011 & 445 & 1,51 & 108 \\
\hline 2012 & 603 & 1,82 & 95 \\
\hline 2013 & 1078 & 2,74 & 81 \\
\hline 2014 & 939 & 2,40 & 81 \\
\hline 2015 & 639 & 1,88 & 94 \\
\hline 2016 & 829 & 2,26 & 87 \\
\hline Moy. décennale années 2010 & 760 & 2 & 83 \\
\hline
\end{tabular}

de 20,7 t/an en 1985 (Jouanneau et al., 1990) est de $15 \%$ supérieure. Des écarts similaires sont constatés pour l'année $1985\left(\mathrm{~F}^{85}{ }_{\text {net }}=11 \mathrm{t} / \mathrm{an}\right.$; Boutier et al., 2000) et pour l'année 1998
$\left(\mathrm{F}^{98}{ }_{\text {net }}=7,4 \mathrm{t} / \mathrm{an}\right.$; Michel et al., 2000). Par défaut, cette comparaison valide le concept de décroissance par décade puisque les données antérieures sont entachées de plus fortes marges d'erreur 


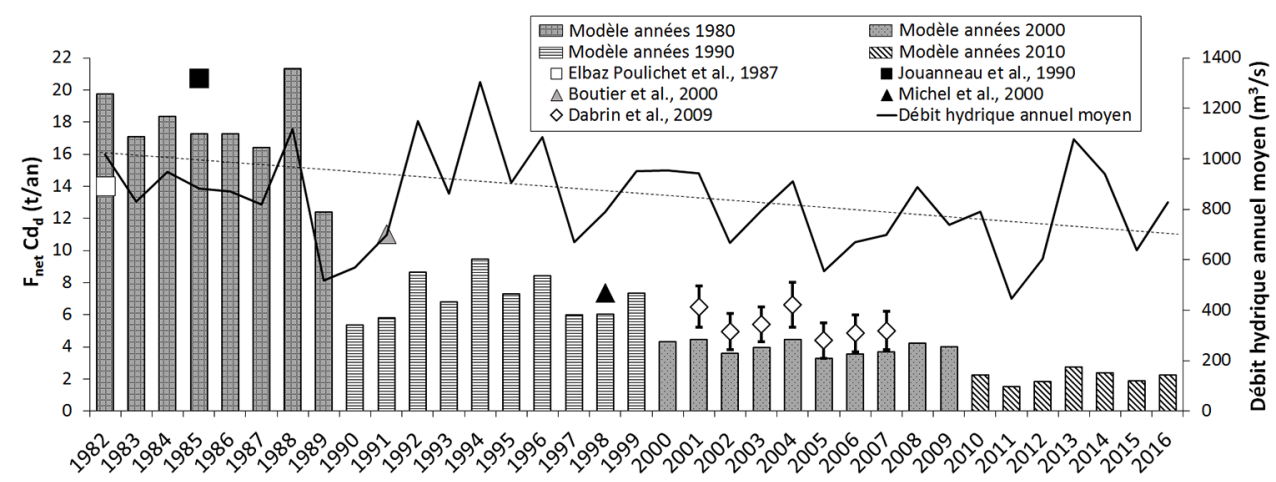

Fig. 7. Les barres grisées et hachurées donnent les variations interannuelles et inter-décennales des flux nets de $\mathrm{Cd}_{d}$ en tonne/an. La variation interannuelle des flux hydriques en $\mathrm{m}^{3} / \mathrm{s}$ est également reportée. Les figurés blancs, noirs et gris correspondent aux valeurs des flux nets publiés antérieurement.

Fig. 7. Grey and hatched vertical bars correspond to the annual and decadal variations of dissolved $\mathrm{Cd}$ net fluxes (t/an). The black, grey and white symbols correspond to previously publish annual $\mathrm{Cd}_{d}$ net flux data. The black line is the annual water discharge $\left(\mathrm{m}^{3} / \mathrm{s}\right)$ and the dashed line indicates its decreasing trend between 1982 and 2016.

mais que les ordres de grandeur de la décontamination du système girondin entre chaque décade sont comparables. Toutefois, les valeurs, reportées entre 1990 et 1994 dans la figure 7, sont certainement sous-estimées. En considérant qu'il existe une certaine proportionnalité entre les $\mathrm{F}_{\text {nets }}^{\text {an }} \mathrm{Cd}_{\mathrm{d}}$ et les concentrations de $\mathrm{Cd}$ accumulées dans le corps mou des huîtres (Fig. 6), cette sous estimation pourrait être de l'ordre de 15 à $25 \%$. L'amplitude de baisse générale des débits sur 26 ans est de l'ordre de $200 \mathrm{~m}^{3} / \mathrm{s}$, dans un contexte de variations interannuelles deux à trois fois supérieures (Fig. 7). Le bilan hydrique régional inclut toutefois, les précipitations pluviales et nivales, l'évapotranspiration et les prélèvements d'eau pour la culture irriguée. La normalisation de $\mathrm{sF}_{\text {nets }}^{\text {an }} \mathrm{Cd}_{\mathrm{d}}$ en t/an par rapport aux débits hydriques annuels en $\mathrm{km}^{3} / \mathrm{an}$ donne des concentrations moyennes pondérées (ng/l) qui montrent que les seuils de décroissance décennale sont conservés (Tab. V). Ainsi, les variations interannuelles de débits ne peuvent être la seule cause des variations constatées des $\mathrm{F}_{\text {nets }}^{\text {an }}$ de $\mathrm{Cd}_{\mathrm{d}}$. De plus, le changement climatique global et ses conséquences régionales ne constituent pas un facteur forçant significatif sur les $\mathrm{F}_{\text {nets }}^{\mathrm{an}}$ en $\mathrm{Cd}_{d}$ et par extension sur les autres polluants métalliques et organiques.

La tendance générale des $F_{\text {nets }}^{\text {an en }}$ $\mathrm{Cd}_{\mathrm{d}}$ depuis 1982 (Fig. 7) montre la nette décroissance de l'exportation de $\mathrm{Cd}_{\mathrm{d}}$ en zone côtière. En effet, le $\mathrm{F}_{\text {net }}^{\mathrm{an}}$ diminue d'environ de moitié par palier décennal avec une moyenne de $F_{\text {net }}^{\text {an }}=17 \mathrm{t}$ /an pour les années 1980, $\mathrm{F}_{\text {net }}^{\mathrm{an}}=7 \mathrm{t}$ /an pour les années 1990, $\mathrm{F}_{\text {net }}^{\mathrm{an}}=4 \mathrm{t} / \mathrm{an}$ pour les années 2000 et $F_{\text {net }}^{\text {an }}=2 \mathrm{t}$ /an pour les années 2010 (Tab. V). L'évolution des $\mathrm{F}_{\text {net }}^{\mathrm{an}}$ de 1982 à 2016 montre ainsi une diminution de l'export de $\mathrm{Cd}_{\mathrm{d}}$ par la Gironde d'environ $85 \%$. Ces résultats sont très 


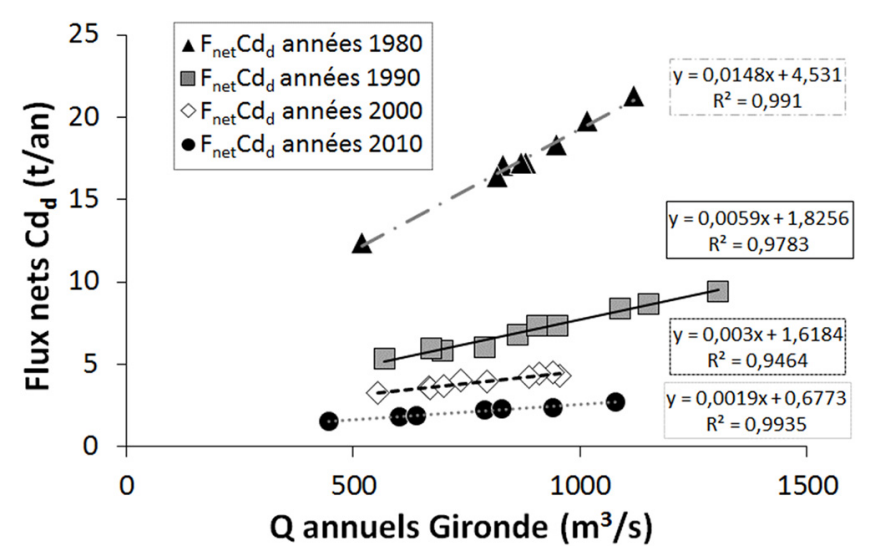

Fig. 8. Diagramme montrant les quatre régressions linéaires servant d'abaques pour l'estimation des flux nets annuels de cadmium dissous $\left(\mathrm{Cd}_{d}\right)$ exportés par la Gironde pour les quatre dernières décades.

Fig. 8. Diagram showing the four linear functions used as a chart to estimate of the annual $\mathrm{Cd}_{d}$ net fluxes over the four last decades.

encourageants et montrent que le temps de résilience des systèmes fluviaux et estuariens dépend majoritairement des investissements consentis à la restauration de bassins versants pollués. La diminution par paliers successifs des flux nets en $\mathrm{Cd}_{d}$ en Gironde est principalement due aux deux grandes étapes de travaux de réhabilitation du site métallurgique de Viviez, après l'arrêt en 1987 des activités industrielles de production de zinc. La conséquence majeure de ces deux étapes de confinement des déchets métallurgiques est une forte baisse des apports de $\mathrm{Cd}$ à la Gironde via le Lot et la Garonne.

Nous devons cependant constater que la dernière réhabilitation (20092017) n'a pas permis aux concentrations en $\mathrm{Cd}$ des huîtres sauvages de la Gironde de passer en dessous de la norme de consommation. Dans l'attente d'une résilience satisfaisante, l'effort d'observation du ROCCH de I'IFREMER devra être poursuivi.
Concernant l'évolution future des $\mathrm{F}_{\text {nets }}^{\text {an }}$ en $\mathrm{Cd}_{\mathrm{d}}$, les données acquises montrent qu'il existe une relation directe entre les $\mathrm{F}_{\text {nets }}^{\mathrm{an}}$ en $\mathrm{Cd}_{d}$ en t/an et les débits hydriques annuels moyens de la Gironde en $\mathrm{m}^{3} / \mathrm{s}$ (Fig. 8). Cette figure montre que les pentes de régression diminuent de 0,006 à 0,003 puis 0,002 , respectivement pour les années 1990, 2000 à 2010 en fonction de la baisse de la pression anthropique. Ainsi, en utilisant le modèle issu du traitement des données acquises en 2014-2015, les flux nets en $\mathrm{Cd}_{d}$ pour l'année 2017 est de 1,7 t/an pour un débit moyen annuel de $527 \mathrm{~m}^{3} / \mathrm{s}$. L'utilisation de cet abaque pour donner dans les prochaines années, les flux nets de $\mathrm{Cd}_{d}$ à partir des seules données de débits moyens annuels de la Gironde sera possible tant que le corps mou des huîtres sauvages montrera des concentrations $\mathrm{Cd}$ comparables à celles mesurées depuis 2010 (Fig. 6). 


\section{Conclusion}

Les données de concentrations de cadmium dissous $\left(\mathrm{Cd}_{\mathrm{d}}\right)$ dans le gradient de salinité estuarien et les concentrations théoriques de Boyle « $\mathrm{Cd}_{d}{ }^{0}$ » acquises en Gironde en 2014 et 2015 en fonction des débits hydriques, ont permis de proposer pour la première fois un modèle empirique analytique pour calculer plus précisément des flux nets annuels de cadmium dissous. Ce modèle a été étendu à la banque de données préexistantes pluri-décennales des " $\mathrm{Cd}_{d}{ }^{0}$ ». Ainsi, la variation interannuelle des flux nets de $\mathrm{Cd}_{d}$ sur les 30 dernières années, proposée dans cette étude intègre les variations des concentrations de Boyle en fonction des débits hydriques, des coefficients de marée et de la diminution par paliers décennaux de la pollution cadmiée. Les flux nets annuels de $\mathrm{Cd}_{d}$ déterminés pour les années 2014 et 2015 sont les plus faibles depuis 30 ans. Ces nouvelles données montrent qu'entre les années 1980 et 2010, les flux de $\mathrm{Cd}_{d}$ exportés à la mer sous forme dissoute dans les années 2010 seraient huit fois plus faibles que ceux exportés dans les années 1980. Toutefois, la baisse de la pollution cadmiée dans le corps mou des huîtres sauvages de la Gironde n'est que d'un facteur cinq (RNO/ $\mathrm{ROCCH}, 2016)$. Ce nouveau modèle confirme une diminution de l'export net de $\mathrm{Cd}$ dissous malgré des variations liées à des conditions de marée et de débits extrêmes ou aux travaux d'aménagement du bassin versant du Lot. Ce modèle valide les effets positifs de la réduction de la source métallurgique suite aux plans de remédiation réalisés sur le site émetteur majoritaire industriel. Cependant, les émissions de $\mathrm{Cd}$ dans le bassin de la Garonne semblent encore trop importantes pour constater une résilience acceptable d'un point de vue sanitaire, de la pollution des huîtres en Gironde. Ce nouveau modèle montre également les limites des modèles numériques précédemment conçus par l'insuffisance de données de terrain et les limites des nombreuses tentatives de calcul de flux sur la base de données de terrain non représentatives des transferts de masse spécifiques du bassin versant étudié. De plus, ce nouveau modèle a permis de proposer un abaque estimant les valeurs futures du flux annuel de cadmium dissous exporté à l'océan en utilisant uniquement les données de débit hydrique annuel des principaux tributaires de l'estuaire de la Gironde. Le lien établi dans cette étude entre la diminution par paliers temporels, des flux nets et des concentrations en $\mathrm{Cd}$ en $\mathrm{mg} / \mathrm{kg}$ p.s. dans le corps mou des huîtres sauvages projette la validité future de l'abaque proposé, jusqu'à la constatation d'un nouveau seuil dégressif des concentrations en $\mathrm{Cd}$ des huîtres de La Fosse (site aval Gironde, réseau RNO-ROCCH de l'Ifremer). Lorsqu'un nouveau seuil sera constaté, il deviendra indispensable de programmer de nouvelles missions océanographiques de prélèvements afin de proposer une nouvelle équation analytique pour déterminer les flux annuels. Au-delà de la diminution des apports de contaminants par la source principale et des effets d'un changement éventuel de régimes hydriques de la 
Garonne dû à des causes climatiques et/ou d'exploitation trop forte de la ressource en eau, les flux nets de $C_{d}$ sont aussi fonction de l'amplitude de l'addition dans le gradient de salinité estuarien. Des investigations portant sur des modifications des réactions de sorption de $\mathrm{Cd}$, seront nécessaires pour quantifier plus précisément la dépollution de l'estuaire en $\mathrm{Cd}$ mais aussi pour étudier les variations de la dynamique estuarienne d'autres polluants métalliques et organiques en fonction de cette dépollution.

\section{REMERCIEMENTS}

Cette étude a été financée par l'Agence de l'Eau Adour Garonne et nous tenons à remercier Jean Pierre Rebillard, Chef de Service Département Connaissance et Système d'Information. Cette étude a reçu le soutien de la Flotte Scientifique Nationale (INSU) et du FEDER via les programmes européens de la Région Aquitaine.

\section{RÉFÉRENCES}

Audry S., 2003. Bilan géochimique du transport des éléments traces métalliques dans le système fluvial anthropisé LotGaronne-Gironde, 413 p. Thèse de Doctorat. Université de Bordeaux 1.

Audry S., Blanc G. \& Schäfer J., 2004a. Cadmium transport in the Lot-Garonne river system (France)-temporal variability and a model for flux estimation, Sci. Total Environ. 319: 197-213.

Audry S., Blanc G. \& Schäfer J., 2004a. Cadmium transport in the LotGaronne River system (France) temporal variability and a model for flux estimation, Sci. Total Environ. 319: 197-213.
Audry S., Schäfer J., Blanc G., Bossy C. \& Lavaux G., 2004b. Anthropogenic components of heavy metal $(\mathrm{Cd}, \mathrm{Zn}$, $\mathrm{Cu}, \mathrm{Pb}$ ) budgets in the Lot-Garonne fluvial system (France), Appl. Geochem. 19: 769-786.

Audry S., Schäfer J., Blanc G. \& Jouanneau J.-M., 2004c. Fifty-year sedimentary record of heavy metal pollution (Cd, $\mathrm{Zn}, \mathrm{Cu}, \mathrm{Pb})$ in the Lot river reservoirs (France), Environ. Pollut. 132: 413-426.

Audry S., Blanc G. \& Schäfer J., 2005. The impact of sulphide oxidation on dissolved metal (Cd, Zn, Cu, Cr, Co, Ni, U) inputs into the Lot-Garonne fluvial system (France), Appl. Geochem. 20: 919-931.

Audry S., Blanc G., Schäfer J., Guérin F., Masson M. \& Robert S., 2007a. Budgets of $\mathrm{Mn}, \mathrm{Cd}$ and $\mathrm{Cu}$ in the macrotidal Gironde estuary (SW France), Mar. Chem. 107: 433-448.

Audry S., Blanc G., Schäfer J. \& Robert S., 2007b. Effect of estuarine sediment resuspension on early diagenesis, sulfide oxidation and dissolved molybdenum and uranium distribution in the Gironde estuary, France, Chem. Geol. 238: 149-167.

Blanc G., Lapaquellerie Y., Maillet N. \& Anschutz P., 1999. A cadmium budget for the Lot-Garonne fluvial system (France). In: Man and River Systems, pp. 331-341. Springer.

Blanc G., Schäfer J., Audry S., Bossy C., Lavaux G. \& Lissalde J.P., 2006. Le cadmium dans le Lot et la Garonne: sources et transport. Hydroéco. Appl. 15: $19-41$.

Blanc G., Schäfer J., Coynel A., Bossy C. \& Dutruch L., 2019. La contamination métallique de l'estuaire de la Gironde. Dans : «Estuaire de la Gironde : état des lieux d'un écosystème sous pression, fragile et vulnérable » Livre blanc de l'estuaire, TH2, Chap.1. Pessac: PUB (Presse Universitaire de Bordeaux) . 
Boyle E.A., Huested S.S. \& Grant B., 1982. The chemical mass balance of the amazon plume-II. Copper, nickel, and cadmium, Deep Sea Res. Part Oceanogr. Res. Pap. 29: 1355-1364.

Boyle E., Collier R., Dengler A., Edmond J., $\mathrm{Ng} \mathrm{A}$. \& Stallard R., 1974. On the chemical mass-balance in estuaries, Geochim. Cosmochim. Acta 38: 1719-1728.

Boutier B. \& Chiffoleau J.-F., 1986. La contamination par le cadmium en Gironde et son extension sur le plateau continental ( ${ }^{\circ}$ DERO-86.12MR). Direction de l'Environnement et des Recherches Océaniques. Plouzané : Département Milieu et Ressources.

Boutier B., Chiffoleau J.-F., Jouanneau J.M., Latouche C. \& Philipps I., 1989. La contamination de la Gironde par le cadmium: origine, extension, importance (Rapport scientifiques et techniques de l'IFREMER N¹4-1989).

Boutier B., Chiffoleau J.-F., Auger D. \& Truquet I. 1993. Influence of the Loire River on dissolved lead and cadmium concentrations in coastal waters of Brittany, Estuar. Coast. Shelf Sci. 36: 133-145.

Boutier B., Chiffoleau J.-F., Gonzalez J.-L., Lazure P., Auger D. \& Truquet I., 2000. Influence of the Gironde estuary outputs on cadmium concentrations in the waters: Consequences on the Marennes-Oléron bay (France), Oceanol. Acta 23: 745-757.

Castaing P. \& Jouanneau J., 1979. Temps de résidence des eaux et des suspensions dans l'estuaire de la Gironde, J. Rech. Océan. IV: 41-52.

Chester R., 1990. The transport of material to the oceans: Relative flux magnitudes, Mar. Geochem, pp.149-191. London: Springer, Academic Division of Unwin Hyman Ltd.
Chiffoleau J.-F., Auger D. \& Chartier E., 1999. Fluxes of selected trace metals from the Seine estuary to the eastern English channel during the period August 1994 to July 1995, Cont. Shelf Res. 19: 2063-2082.

Chiffoleau J.-F., Cossa D., Auger D. \& Truquet I., 1994. Trace metal distribution, partition and fluxes in the Seine estuary (France) in low discharge regime, Mar. Chem. 47: 145-158.

Chiffoleau J.-F., Auger D., Chartier E., Michel P., Truquet I., Ficht A., Gonzalez J.-L. \& Romana L.-A. 2001. Spatiotemporal changes in cadmium contamination in the Seine estuary (France), Estuaries 24: 1029-1040.

Claisse D., Joanny M. \& Quintin J.-Y., 1992. Le réseau national d'observation de la qualité du milieu marin (RNO), Analusis: M19-M22.

Comans R.N. \& van Dijk C.P., 1988. Role of complexation processes in cadmium mobilization during estuarine mixing, Nature 336: 151-154.

Dabrin A., Schäfer J., Blanc G., Strady E., Masson M., Bossy C., Castelle S., Girardot N. \& Coynel A., 2009. Improving estuarine net flux estimates for dissolved cadmium export at the annual timescale: Application to the Gironde Estuary, Estuar. Coast. Shelf Sci. 84: 429-439.

Dabrin A., Schäfer J., Bertrand O., Masson M. \& Blanc G., 2014. Origin of suspended matter and sediment inferred from the residual metal fraction: Application to the Marennes Oleron Bay, France, Cont. Shelf Res. 72: 119-130.

Directive 2013/39/UE du Parlement Européen et du Conseil du 12 août 2013 modifiant les directives 2000/60/CE et 2008/105/CE en ce qui concerne les substances prioritaires pour la politique dans le domaine de l'eau. 
Edmond J.M., Spivack A., Grant B.C., Ming-Hui H., Zexiam C., Sung C. \& Xiushau Z., 1985. Chemical dynamics of the Changjiang estuary, Cont. Shelf Res. 4: 17-36.

Elbaz-Poulichet F., Martin J., Huang W. \& Zhu J., 1987. Dissolved Cd behaviour in some selected French and Chinese estuaries. Consequences on Cd supply to the ocean, Mar. Chem. 22: 125-136.

Grousset F.E., Jouanneau J.M., Castaing P., Lavaux G. \& Latouche C., 1999. A 70 year record of contamination from industrial activity along the Garonne river and its tributaries (SW France), Estuar. Coast. Shelf Sci. 48: 401-414.

Jouanneau J.M., Boutier B., Chiffoleau J.F., Latouche C. \& Philipps I., 1990. Cadmium in the Gironde fluvioestuarine system: Behaviour and flow, Sci. Total Environ. 97: 465-479.

Kraepiel A.M., Chiffoleau J.-F., Martin J.-M. \& Morel F.M., 1997. Geochemistry of trace metals in the Gironde estuary. Geochim. Cosmochim. Acta 61: 14211436.

Lanceleur L., Schäfer J., Bossy C., Coynel A., Larrose A., Masson M. \& Blanc G., 2011a. Silver fluxes to the Gironde estuary-Eleven years (1999-2009) of monitoring at the watershed scale, Appl. Geochem. 26: 797-808.

Lanceleur L., Schäfer J., Chiffoleau J.F., Audry S., Auger D., Renault S., Baudrimont M. \& Blanc G., 2011b. Longterm (30 years) records and relationships of cadmium and silver contamination in sediment and oysters from the Gironde fluvial-estuarine continuum, Chemosphere 85: 1299-1305.

Larrose A., Coynel A., Schäfer J., Blanc G., Massé L. \& Maneux E., 2010. Assessing the current state of the Gironde Estuary by mapping priority contaminant distribution and risk potential in surface sediment, Appl. Geochem. 25: 1912-1923.
Latouche C., 1988. Cadmium pollution in the Gironde estuary, Bull. L'institut Géologique Bassin D'Aquitaine Bordx 44: 15-21.

Mantoura R., 1981. Organo-metallic interactions in natural waters, Elsevier Oceanogr. Ser. 31: 179-223.

Mantoura R., Dickson A. \& Riley J., 1978. The complexation of metals with humic materials in natural waters, Estuar. Coast. Mar. Sci. 6: 387-408.

Masson M., Blanc G. \& Schäfer J., 2006. Geochemical signals and source contributions to heavy metal $(\mathrm{Cd}, \mathrm{Zn}, \mathrm{Pb}, \mathrm{Cu})$ fluxes into the Gironde Estuary via its major tributaries, Sci. Total Environ. 370: 133-146.

Michel P., Boutier B. \& Chiffoleau J.-F., 2000. Net fluxes of dissolved arsenic, cadmium, copper, zinc, nitrogen and phosphorus from the Gironde estuary (France): Seasonal variations and trends, Estuar. Coast. Shelf Sci. 51: 451-462.

$\mathrm{RNO} / \mathrm{ROCCH}$, 2016. Qualité du Milieu Marin Littoral Bulletin de la surveillance 2016. Départements: Gironde, Landes, Pyrénées Atlantiques. Océanographie et Dynamique des Ecosystèmes Unité Littoral Laboratoire Environnement Ressources d'Arcachon. Bulletin de surveillance. Juin 2017-ODE/LITTORAL/LERAR/17-004.

Robert S., Blanc G., Schäfer J., Lavaux G. \& Abril G., 2004. Metal mobilization in the Gironde Estuary (France): The role of the soft mud layer in the maximum turbidity zone, Mar. Chem. 87: 1-13.

Schäfer J. \& Blanc G., 2002. Relationship between ore deposits in river catchments and geochemistry of suspended particulate matter from six rivers in southwest France, Sci. Total Environ. 298: 103-118.

Schäfer J., Blanc G., Bossy C., Guérin F., Lapaquellerie Y., Lavaux G., Lissalde J. P., Masson M., Maillet N. \& Robert S., 2002. Budget of the metal inputs into the 
Gironde estuary: Cd desorption process in the salinity gradient (Bilan des apports métalliques à l'estuaire de la Gironde: processus de désorption de cadmium dans le gradient de salinité), Final Sci. Rep. Liteau Program.

Strady E., 2010. Mécanismes biogéochimiques de la contamination des huîtres Crassostrea gigas en Cadmium en baie de Marennes Oléron, 251p. Thèse de Doctorat. Université de Bordeaux 1.

Strady E., Blanc G., Schäfer J., Coynel A. \& Dabrin A., 2009. Dissolved uranium, vanadium and molybdenum behaviours during contrasting freshwater discharges in the Gironde estuary
(SW France), Estuar. Coast. Shelf Sci. 83: 550-560.

Strady E., Blanc G., Baudrimont M., Schäfer J., Robert S. \& Lafon V., 2011a. Roles of regional hydrodynamic and trophic contamination in cadmium bioaccumulation by Pacific oysters in the Marennes-Oléron Bay (France), Chemosphere 84: 80-90.

Strady E., Kervella S., Blanc G., Robert S., Stanisière J.Y., Coynel A. \& Schäfer J., 2011b. Spatial and temporal variations in trace metal concentrations in surface sediments of the Marennes Oléron Bay. Relation to hydrodynamic forcing, Cont. Shelf Res. 31: 997-1007. 